\title{
SOLAR MULTI-CONJUGATE ADAPTIVE OPTICS AT THE DUNN SOLAR TELESCOPE
}

\author{
T. Rimmele ${ }^{1, a}$, S. Hegwer ${ }^{1}$, J. Marino ${ }^{2}$, K. Richards ${ }^{1}$, D. Schmidt ${ }^{3}$, T. Waldmann ${ }^{3}$, and F. Woeger ${ }^{1}$ \\ 1 National Solar Observatory, Sunspot, NM-88349, USA \\ 2 University of Florida, Gainesville, FL \\ 3 Kiepenheuer Institute, Freiburg, Germany
}

\begin{abstract}
Solar observations are performed over an extended field of view and the isoplanatic patch over which conventional adaptive optics (AO) provides diffraction limited resolution is a severe limitation. The development of multi-conjugate adaptive optics (MCAO) for the next generation large aperture solar telescopes is thus a top priority. The Sun is an ideal object for the development of MCAO since solar structure provides ,,multiple guide stars" in any desired configuration. At the Dunn Solar Telescope (DST) we implemented a dedicated MCAO bench with the goal of developing well-characterized, operational MCAO. The MCAO system uses 2 deformable mirrors conjugated to the telescope entrance pupil and a layer in the upper atmosphere, respectively. DM2 can be placed at conjugates ranging from $2 \mathrm{~km}$ to $10 \mathrm{~km}$ altitude. We have successfully and stably locked the MCAO system on artificial objects (slides), for which turbulence screens are generated directly in front of the DMs, as well as solar structure. We present preliminary results and discuss future plans.
\end{abstract}

\section{Introduction}

Solar adaptive optics has become an indispensable tool at ground based solar telescopes. Driven by the quest for ever higher spatial resolution observations of the Sun solar adaptive optics are now operated routinely at major ground based solar telescopes. The current high-resolution solar telescopes, such as the Dunn Solar Telescope (DST), are in the one-meter class and utilize AO for $\geq 95 \%$ of the observing time to achieve the diffraction limit at visible and NIR wavelengths. Solar AO [1,2] has revitalized ground-based solar astronomy at existing telescopes. The development of high-order solar AO that is capable of delivering high Strehl in the visible will be absolutely essential for next generation solar telescopes, such as the $4 \mathrm{~m}$ aperture Advanced Technology Solar Telescope (ATST), which undoubtedly will revolutionize solar astronomy [3].

Solar observations are performed over an extended field of view. The limited size of the isoplanatic patch, over which conventional adaptive optics (AO) provides diffraction limited resolution is often a severe limitation. Figure 1 (left) shows an image of solar granulation and embedded magnetic g-band bright points observed near the limb of the sun. The field of view is approximately 120 "x 80 ". This diffraction limited image was recorded at the Dunn Solar Telescope with high order adaptive optics and post-processed using speckle interferometry [4]. Post-processing is required to achieve the uniform, diffraction limited imaging over such an extended FOV. However, speckle interferometry as well as other post facto restoration methods typically rely on short exposure imaging, which in most cases can not be deployed when quantitative spectroscopy and polarimetry is performed, i.e., long exposures are required.

In the visible, conventional AO limits the corrected FOV to about $10^{\prime \prime}$ or less. In contrast, a sunspot or active region extends typically over 1-2 arcmin. In order to capture the dynamic temporal evolution of sunspot structures on the relevant spatial scales, one requires spatial resolution of $0^{\prime \prime} .1$ or less. Flares can occur "unannounced" anywhere in the extended FOV. Flare trigger mechanisms operate rapidly and on the smallest spatial scales. Their location within the FOV is difficult, if not impossible to predict. Thus, we are led directly to a requirement for diffraction limited resolution over a FOV of 1-2 arcmin. Multi-conjugate adaptive optics (MCAO) is a technique that provides real-time

a e-mail: rimmele@nso.edu

This is an Open Access article distributed under the terms of the Creative Commons Attribution-Noncommercial License, which permits unrestricted use, distribution, and reproduction in any noncommercial medium, provided the original work is properly cited. 
diffraction limited imaging over an extended FOV [5]. MCAO will eliminate a major limitation of conventional AO systems, the small isoplanatic patch, and, thus provide the tool to effectively study, with unprecedented spatial resolution, fundamental scientific questions like the onset and evolution of flares, flare triggering mechanisms, magnetic reconnection events and many other dynamic solar phenomena that require diffraction limited observations over an extended FOV with the requisite high temporal cadence.

The development of MCAO for existing solar telescopes and, in particular, for the next generation large aperture solar telescopes is thus a top priority. The Sun is an ideal object for the development of MCAO since solar structure provides "multiple guide stars" in any desired configuration. It is therefore not surprising that the first successful on-the-sky MCAO experiments were performed at the Dunn Solar Telescope and at a solar telescope on the Canary Islands. However, further development is needed before operational solar MCAO can be implemented at future large aperture solar telescopes such as the ATST on Haleakala [6]. MCAO development must progress beyond these initial proof-of-concept experiments and should include laboratory experiments and on-sky demonstrations under controlled or well characterized conditions as well as quantitative performance analysis and comparison to model predictions. The MCAO effort at the DST described in this paper is a significant step in this direction.
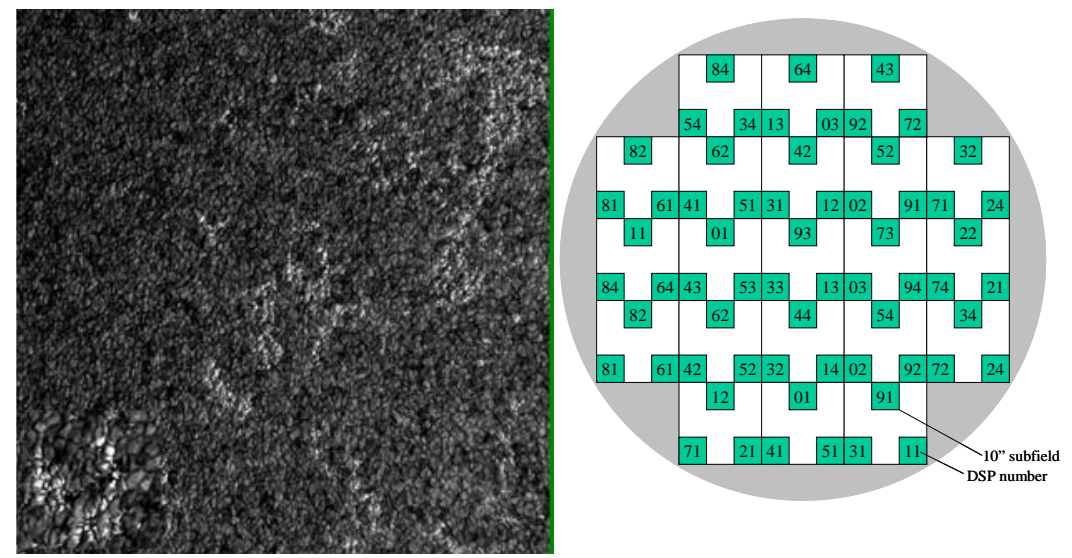

Fig. 1. Left: Image of solar granulation with g-band bright points observed near the limb of the sun. The bright points (see zoomed in insert in the lower left corner) are caused by strong magnetic field concentrations. The field of view is approximately 120"x 80". Right: MCAO WFS geometry. Each of the square subimages contains a 52"x52" image of granulation as seen on the left. The wavefront sensor camera of the MCAO stage reads out three 20x20 pixel (10"x10") fields arranged in a triangular pattern from within a 52"x52" FOV. The separation between guide regions can be adjusted by simply reprogramming the WFS camera. For the experiment described here the diagonal distance between the centers of the guide regions was about $30 \mathrm{arcsec}$.

\section{PREVIOUS MCAO EXPERIMENTS AND LIMITATIONS}

The initial solar MCAO work performed at NSO [7,8] and at the Kiepenheuer Institute [9] provided proof-of-concept with on-sky experiments at the DST and the German VTT on the Canaries. Both experiments demonstrated the MCAO's ability to extend the corrected FOV. The DST MCAO experiments used two DMs and a MCAO wavefront sensor with three guide regions. Lacking dedicated hardware these experiments utilized existing hardware from the DST conventional AO systems. Due 
to the constraints given by the existing hardware only three guide regions and a limited number of subapertures ( 21 per guide region) could be implemented at a reasonable bandwidth. However, since day-time seeing is often dominated by strong ground-layer turbulence a high order correction of the ground-layer is crucial. The 76 subaperture conventional AO system at the $76 \mathrm{~cm}$ DST can correct between 60 and $70 \mathrm{KL}$ modes and thus is able to provide good correction for most seeing conditions. By limiting the ground layer correction to only 21 subapertures MCAO work was practically limited to the very best seeing conditions only.

Another severe practical limitation was the fact that in order to set up the MCAO experiment the two operational conventional AO systems had to be disassembled to provide the parts such as DMs and wavefront sensor equipment for the MCAO. Likewise, a complete and time consuming re-assembly and alignment of the conventional AO systems was necessary after completion of the MCAO experiment. Given the high demand for observing time at the DST this situation was no longer sustainable.

The above mentioned on-sky experiments were performed under unknown atmospheric conditions, i.e., no information about the $\mathrm{Cn} 2(\mathrm{~h})$ profile above the telescope was available during the experiments. Hence, a detailed comparison of MCAO performance with model predictions was impossible. Furthermore, optimization of parameters such as DM conjugate positions and control algorithms could not be performed. However, before any operational solar MCAO can be designed or built it is crucial to determine how well achieved and expected performance compare and under what conditions and operational parameters.

\section{MODIFIED MCAO APPROACH AND DESIGN}

At the DST we have now implement a dedicated MCAO bench allows for easier and faster and somewhat more permanent implementation of the MCAO experiment. In order to achieve this goal we implemented a staged approach that is uniquely applicable to solar MCAO. The first stage consists of the high order AO system with 76 subapertures and a 97 actuator DM conjugated to ground level. This system is used for normal operations at the DST. The second stage uses a second 97 actuator DM that can be conjugated to an adjustable altitude between 2 and $10 \mathrm{~km}$ [14]. The wavefront sensor uses 3-4 off-axis guide regions to track granulation or other solar structure.

The wavefront sensor of the first stage, high order $\mathrm{AO}$ was modified by replacing the lenslet array to provide a wide field of $\geq 20$ " $x 20$ " for correlation tracking. The conventional AO uses a small FOV of about 8 "x8" and thus provides high order correction more or less on-axis. By computing cross correlations of a large FOV we obtain wavefront aberrations averaged over many field angles and several isoplanatic patch sizes, respectively, effectively providing the desired high order ground layer correction with this first stage of the MCAO system [10]. The wavefront sensor of the second stage (WFS2) that follows the ground-layer AO utilizes 3-4 guide regions of about 10"x10" each. The triangular arrangement of the 3 guide-regions setup is shown in figure 1 (right). The spare wavefront sensor camera and processing unit of the high order AO system was used for the second stage of the MCAO experiment. The camera can easily be reprogrammed to accommodate the arrangement of multiple FOVs shown in figure 1. The DSP processing unit is used to process 3-4 guide regions with 21 subapertures each. The system runs at $1.6 \mathrm{kHz}$ frame rate. In principle, any number of subfields can be placed within the large FOV in any desired geometrical arrangement. In practice, the number of guide regions is limited by the processing power of the DSP unit. Reconstruction matrices that compute actuator commands from the WFS measurements are constructed by generating a modal interaction matrix and applying a standard Singular Value Decomposition (SVD) algorithm to compute the pseudo inverse. The number of KL modes that can be corrected with DM2 in a stable control loop depends on the seeing conditions and ranges between 15-20 modes. It is expected that a more sophisticated reconstruction techniques with proper regularization applied would allow us to control additional modes. This will be investigated in experiments that will be performed in the near futures. Another interesting and unusual feature of this approach is that the control loops for DM1 and DM2 are entirely separated, which results in increased robustness of the MCAO system. Once the loop is closed on the high order ground-layer AO WFS2 measures aberrations caused by the high altitude seeing only. 


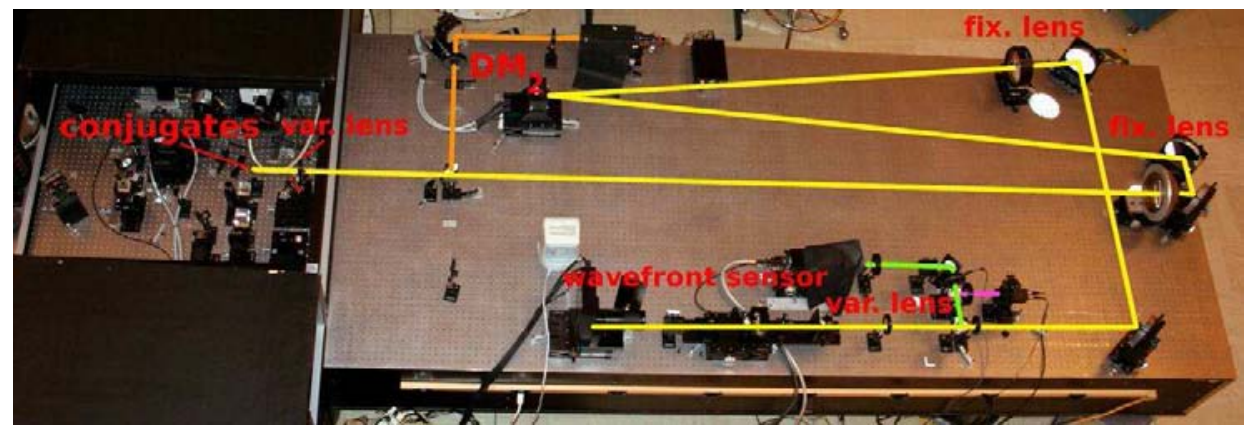

Fig. 2. Optical setup

\section{TURBULENCE PROFILE ESTIMATION}

One of the main goals of the DST MCAO experiments is to perform detailed on-sky evaluations and comparison to simulated performance estimations. A crucial ingredient to this effort is knowledge of the turbulence profile above the DST at the time the experiments are performed. A method to determine $\mathrm{Cn} 2(\mathrm{~h})$ that uses an extended field Shack-Hartmann WFS and is similar to the night-time SLODAR [11] is being developed [12] and will be implemented at the DST. The ultimate goal is to provide close to real-time turbulence profiling. The measurements we performed on Aug. 13, 2008 are representative for an observing day at Sac Peak during which seeing conditions are excellent. The real time video images indicated that there was little high altitude $(\geq 10 \mathrm{~km})$ turbulence caused by the jet stream dipping far to the south as it is typically present during less favorable seeing conditions. These measurements are consistent with a turbulence layer located between $2 \mathrm{~km}$ and $4 \mathrm{~km}$ above the telescope [14]. Solar observations are usually performed in the morning hours and ideally the altitude of the conjugate for DM2 should be adjustable on short time scales. This is not practical with the setup shown in figure 2. As a compromise we chose a conjugate altitude of $4 \mathrm{~km}$ for DM2. Detailed simulations will be used to optimize the location of DM2.

\section{RESULTS}

The observations were performed during May and Aug. 2008. Bursts of 50 - 100 digital 1kx1k images with an exposure time of $10 \mathrm{~ms}$ were recorded after ground-layer AO correction and after the MCAO correction, respectively. The images were recorded strictly simultaneously at a frame rate of $8 \mathrm{~Hz}$. The observing wavelength was $510 \mathrm{~nm}(9 \mathrm{~nm}$ FWHM) for both channels. During these runs DM2 was positioned at conjugate heights of $2,4,5$ and $7 \mathrm{~km}$. The best results were achieved with the DM2 at 4 and $5 \mathrm{~km}$ conjugates, which is not surprising given the results of the turbulence profiling [14] obtained during the Aug. 2008 period. The number of controlled modes was varied between 9 and $20 \mathrm{KL}$ modes. The control loop quickly became unstable during attempts to control more modes. The image bursts were processed in two ways. A local correlation algorithm was applied to obtain residual image motion within the extended FOV of about 60" diameter. Residual image motion is easily computed from granulation images and is an indicator for the degree of correction obtained. Alternatively, a speckle reconstruction algorithm [13] was applied, which also outputs residual image motion. The results of both methods are nearly identical.

Figure 3 shows two examples of residual image motion maps pairs. The granulation image in the background is the speckle reconstruction of the corresponding burst of images. Dark areas in these images indicate good correction. The top images are recorded after DM2 and demonstrate the ability of the MCAO stage to provide an increased corrected FOV compared to the conventional AO. DM2 corrected $20 \mathrm{KL}$ modes for these cases. The residual image motion maps recorded after DM1 (bottom) are not uniform across the FOV indicating that our wide field wavefront sensor approach did not deliver 

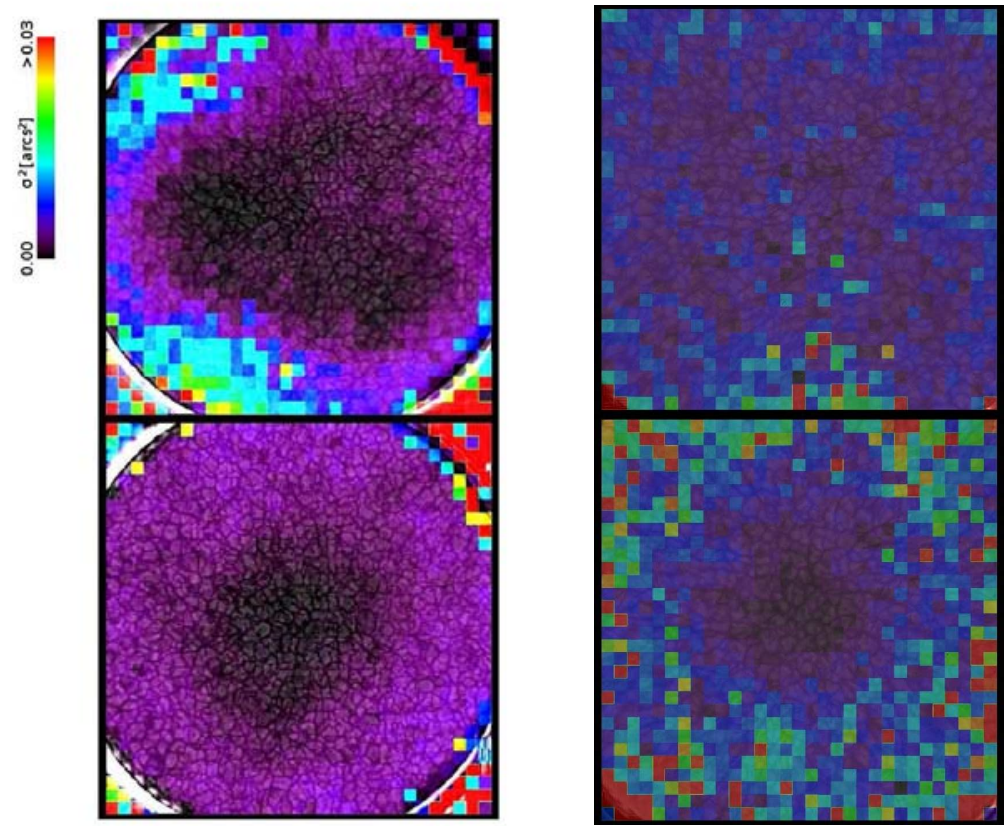

Fig. 3. Maps of residual image motion measured with conventional AO (bottom) and the MCAO (top). Dark areas in these images indicate good correction. The circular FOV is about 1 arcmin. The example on the left was obtained using the three guide region configuration shown in Figure 1. The example on the right was obtained with four guide stars. The square FOV is about 45 " $x 45$ " and a different color scaling has been applied to the residual image motion maps. Both examples clearly demonstrate the MCAO's capability to increase the corrected FOV.

a perfect ground-layer correction but still corrected more efficiently on-axis. The MCAO corrects to a level of less than 0".01 rms and within a triangular shaped FOV of about 40" compared to typically less than 10 " of the conventional AO. The location of the guide regions is clearly reflected in the residual image motion maps since the best correction is at these locations. Image motion is actually increased by the MCAO stage in the corners of the FOV where the 3 guide-region wavefront sensor does not provide any information.

\section{CONCLUSIONS AND FUTURE WORK}

We presented results from our solar MCAO experiment performed at the DST. Our approach, which combines a wide field correlating Shack Hartmann AO system with a 3-4 guide-region MCAO stage is somewhat unusual and uniquely applicable to solar MCAO and possibly other extended object AO systems. At the DST this approach allows us to quickly switch from normal operations with conventional AO to the experimental MCAO development work and, in addition, provides without any new hardware required the high order correction of the strong ground-layer, which is dominant during day-typical time seeing. We demonstrated that this approach provides a corrected FOV 3-4 times that of the conventional AO. Future efforts will compare the performance of the two-staged approach with that of a MCAO approach that uses a generalized interaction matrix approach and a global control loop. Simulations indicate that slightly better performance can be achieved with such an approach depending on turbulence distribution and the specific FOV chosen for the wavefront sensor(s). The goal of the MCAO effort at the DST is to develop this MCAO technology for large aperture solar telescopes such as the BBSO NST and the ATST. 


\section{References}

1. Rimmele, T. R., "Recent Advances in Solar Adaptive Optics", 2004, SPIE 5490, 34

2. Rimmele, T. R.: "Solar Adaptive Optics: Conventional and Multi-Conjugate", Proc. of the 2005 AMOS Technical Conference (Maui Economic Development Board), pp. 575

3. Rimmele, T.; Keil, S.; Wagner, J., 2006, "The unique scientific capabilities of the Advanced Technology Solar Telescope", 36th COSPAR Scientific Assembly. Held 16 - 23 July 2004, in Beijing, China., p.3186

4. Wöger, Friedrich; von der Lühe, Oskar, "KISIP: a software package for speckle interferometry of adaptive optics corrected solar data",Advanced Software and Control for Astronomy II. Edited by Bridger, Alan; Radziwill, Nicole M. Proceedings of the SPIE, Volume 7019, pp. 70191E-70191E-8 (2008).

5. Beckers, J. M 1988, "Increasing the Size of the Isoplanatic Patch with Multiconjugate Adaptive Optics", in: Very Large Telescopes and their Instrumentation, ESO Conference and Workshop Proceedings, Proceedings of a ESO Conference on Very Large Telescopes and their Instrumentation, held in Garching, March 21-24, 1988, Garching: European Southern Observatory (ESO), edited by Marie-Helene Ulrich., p.693

6. Rimmele, T., Richards, K., Roche, J.M., Hegwer, S.L., Hubbard, R.P., Hansen, E.R., Goodrich, B., Upton, R.S. "The wavefront correction system for the Advanced Technology Solar Telescope." Advances in Adaptive Optics II., Edited by Ellerbroek, Brent L.; Bonaccini Calia, Domenico., Proceedings of the SPIE, Volume 6272, pp., 627212 (2006).

7. Langlois, M., Moretto, G., Richards, C., Rimmele, T., Hegwer, S., SPIE 5490, 59

8. Rimmele, Thomas; Richards, Kit; Roche, Jacqueline; Hegwer, Steve; Tritschler, Alexandra, 2006, "Progress with solar multi-conjugate adaptive optics at NSO", SPIE, 6272, 5

9. Berkefeld, Thomas; Soltau, Dirk; von der Luehe, Oskar, 2003, "Multi-conjugate Adaptive Optics at the Vacuum Tower Telescope, Tenerife",in: Adaptive Optical System Technologies II. Edited by Wizinowich, Peter L.; Bonaccini, Domenico. Proceedings of the SPIE 4839, pp. 544-553

10. Woeger,F., Rimmele,T., 2008, Applied Optics, vol. 48, issue 1, p. A35

11. Torsten A. Waldmann, Thomas Berkefeld, and Oskar von der Luehe. Turbulence height profiling using wide field of view Hartmann-Shack wavefront sensors, Proc. of the SPIE, 2008

12. Wilson, R.W., "SLODAR: measuring optical turbulence altitude with a Shack-Hartmann wavefront sensor." Mon. Not. R. Astron. Soc. 337, 2002.

13. Woeger, F., von der Luehe, O. , Reardon, K. , "Speckle interferometry with adaptive optics corrected solar data", 2008, A\&A, 488, 375

14. 103. Rimmele, T.; Hegwer, S.; Richards, K.; Woeger, F, Schmidt, D., Waldmann, T. , "Solar Multi-Conjugate Adaptive Optics at the Dunn Solar Telescope", Proceedings of the Advanced Maui Optical and Space Surveillance Technologies Conference, held in Wailea, Maui, Hawaii, September 17-19, 2008, Ed.: S. Ryan, The Maui Economic Development Board., p.E18, (2008) 